PROCEEDINGS OF THE

AMERICAN MATHEMATICAL SOCIETY

Volume 125, Number 10, October 1997, Pages 2847-2851

S 0002-9939(97)03988-9

\title{
A COMMUTING PAIR IN HOPF ALGEBRAS
}

\author{
YONGCHANG ZHU
}

(Communicated by Ken Goodearl)

\begin{abstract}
We prove that if $H$ is a semisimple Hopf algebra, then the action of the Drinfeld double $D(H)$ on $H$ and the action of the character algebra on $H$ form a commuting pair. This result and a result of G. I. Kats imply that the dimension of every simple $D(H)$-submodule of $H$ is a divisor of $\operatorname{dim}(H)$.
\end{abstract}

Let $H$ be a finite dimensional semisimple Hopf algebra over an algebraically closed field $k$ of characteristic $0, D(H)$ be the Drinfeld double of $H$, and $C(H)$ be the character algebra of $H . C(H)$ is spanned by the characters of $H$-modules and is an associative subalgebra of $H^{*}$. It is known that $D(H)$ acts on $H$ and that $C(H)$ acts on $H$ by the restriction of the action "- - of $H^{*}$ on $H$ (these actions will be recalled below). The purpose of this note is to prove that these two actions form a commuting pair. Using this result, we prove that the dimension of every simple $D(H)$-submodule of $H$ is a divisor of $\operatorname{dim}(H)$. It would be interesting if there exists an analog of this commuting pair in the context of Poisson Lie groups.

We first recall the construction of the Drinfeld double (cf. [D], [M]) and fix necessary notations. Let $H$ be a finite dimensional Hopf algebra over a field $k$ (here we do not need any additional assumptions on $H$ and $k$ ). The Drinfeld double of $H$, denoted by $D(H)$, as a vector space, is the tensor space $H^{*} \otimes H$. The comultiplication of $D(A)$ is given by

$$
\Delta(f \otimes a)=\sum\left(f_{(2)} \otimes a_{(1)}\right) \otimes\left(f_{(1)} \otimes a_{(2)}\right) \in D(H) \otimes D(H),
$$

where $\Delta f=f_{(1)} \otimes f_{(2)}, \Delta a=a_{(1)} \otimes a_{(2)}$ are comultiplictions in $H$ and $H^{*}$ respectively. The multiplication in $D(H)$ is defined as follows: for $f \otimes a$ and $g \otimes b$ in $D(H)$,

$$
(f \otimes a)(g \otimes b)=\sum f\left(a_{(1)} \triangleright g_{(2)}\right) \otimes\left(a_{(2)} \triangleleft g_{(1)}\right) b,
$$

where $a \triangleright g$ is the action of $H$ on $H^{*}$ given by

$$
a \triangleright g=a_{(1)} \rightarrow g<S^{-1} a_{(2)}
$$

and $a \triangleleft g$ is the right action of $H^{*}$ on $H$ given by

$$
a \triangleleft g=S^{-1} g_{(1)} \rightarrow a<g_{(2)} .
$$

The notations $\rightarrow$ and $\leftarrow$ mean the usual left and right actions of $H$ on $H^{*}$, i.e., for $a \in H$ and $g \in H^{*}$,

$$
a \rightarrow g=\sum g_{(1)}\left\langle g_{(2)}, a\right\rangle \in H^{*}, \quad g \leftarrow a=\sum g_{(2)}\left\langle g_{(1)}, a\right\rangle .
$$

Received by the editors December 6, 1995 and, in revised form, April 30, 1996. 1991 Mathematics Subject Classification. Primary 16W30. 
We view $H$ and $H^{*}$ as subspaces of $D(H)$ by the embeddings $a \in H \mapsto 1 \otimes a$ and $f \in H^{*} \mapsto f \otimes 1$.

The antipode $S$ and the counit of $D(H)$ are given by

$$
S(f \otimes a)=S(a) S^{-1}(f), \quad \epsilon(f \otimes a)=\epsilon(f) \epsilon(a) ;
$$

here $\epsilon(f)$ and $\epsilon(a)$ denote the counit maps for $H^{*}$ and $H$.

The above operations give $D(H)$ a structure of Hopf algebra. Moreover $D(H)$ is quasitriangular with the $R$-matrix $R=\sum_{i} e_{i}^{*} \otimes e_{i}$, where $\left\{e_{i}\right\}$ is a basis for $H$, and $\left\{e_{i}^{*}\right\}$ is its dual basis for $H^{*}$. The quasitriangular structure will not play a role here. Notice that $H$ and $H^{* c o p}$ ( $H^{* c o p}$ is $H^{*}$ with the opposite coproduct) are Hopf subalgebras of $D(H)$.

We also recall some basic notions about modules and module algebras of a Hopf algebra. A (left) module of $H$ means a left module of $H$ as an associative algebra. An associative algebra $A$ is called a (left) module algebra of $H$ if $A$ is a $H$-module such that the algebra structure and $H$-module structure for $A$ are compatible in the following sense: for $h \in H, u, v \in A$ and the unit $1_{A}$ in $A$,

$$
h \cdot(u v)=\sum\left(h_{(1)} \cdot u\right)\left(h_{(2)} \cdot v\right), \quad h \cdot 1_{A}=\epsilon(h) 1_{A} .
$$

Similarly a right module algebra of $H$ is an associative algebra $A$ together with a right $H$-module structure satisfying the conditions

$$
(u v) \cdot h=\left(u \cdot h_{(1)}\right)\left(v \cdot h_{(2)}\right), \quad 1_{A} \cdot h=\epsilon(h) 1_{A} .
$$

For a finite dimensional $H$-module $V$, the character $\chi_{V}$ of $V$ is an element of $H^{*}$ defined by $\left\langle\chi_{V}, a\right\rangle=\left.T r\right|_{V}(a)$ for every $a \in H$. Because $\chi_{W \otimes V}=\chi_{W} \chi_{V}$ for $H$-modules $W, V$, the characters of $H$ span an associative subalgebra of $H^{*}$. This algebra is called the character algebra of $H$ and denoted by $C(H)$. If $H$ is semisimple and the ground field is algebraically closed and of characteristic 0 , then $C(H)$ consists of the elements $v \in H^{*}$ that are cocommutative, i.e., $\sum v_{(1)} \otimes v_{(2)}=$ $\sum v_{(2)} \otimes v_{(1)}$

$H$ is an $H^{*}$-module algebra under the action $\rightarrow$ given by $g \rightarrow a=\left\langle g, a_{(2)}\right\rangle a_{(1)}$. We will be concerned with the restriction of $\rightarrow$ on the character algebra $C(H)$.

There is $D(H)$-action on $H$ defined by

$$
(f \otimes a) \cdot b=\left(a_{(1)} b S\left(a_{(2)}\right)\right)<S^{-1}(f) .
$$

Lemma 1. $H$ is a module algebra of $D(H)$ under the action (3).

Proof. To prove $H$ is a $D(H)$-module under (3), We need to prove that

$$
(x y) \cdot v=x \cdot(y \cdot v)
$$

for every $x, y \in D(H)$ and $v \in H$. This is true for the cases $x, y \in H \subset D(H)$, $x, y \in H^{*} \subset D(H)$ and $x \in H^{*}, y \in H$. It is known that the definition of the multiplication of $D(H)$ is equivalent to the following (cf. [M])

$$
(f \otimes a)(g \otimes b)=f\left(a_{(1)} \rightarrow g \leftarrow S^{-1} a_{(3)}\right) \otimes a_{(2)} b .
$$

To prove (3), we only need to prove for $a \in H \subset D(H), g \in H^{*} \subset D(H)$ and $v \in H$,

$$
a \cdot(g \cdot v)=(a g) \cdot v=\left(a_{(1)} \rightarrow g \leftarrow S^{-1} a_{(3)}\right) \cdot\left(a_{(2)} \cdot v\right) .
$$


This is proved in the following computation:

$$
\begin{aligned}
& \left(a_{(1)} \rightarrow g-S^{-1} a_{(3)}\right) \cdot\left(a_{(2)} \cdot v\right) \\
& =\left\langle g_{(3)}, a_{(1)}\right\rangle\left\langle g_{(1)}, S^{-1} a_{(3)}\right\rangle g_{(2)} \cdot\left(a_{(2)} \cdot v\right) \\
& =\left\langle g_{(3)}, a_{(1)}\right\rangle\left\langle g_{(1)}, S^{-1} a_{(4)}\right\rangle g_{(2)} \cdot\left(a_{(2)} v S a_{(3)}\right) \\
& =\left\langle g_{(3)}, a_{(1)}\right\rangle\left\langle g_{(1)}, S^{-1} a_{(4)}\right\rangle\left(a_{(2)} v S a_{(3)}\right) \leftarrow S^{-1} g_{(2)} \\
& =\left\langle g_{(3)}, a_{(1)}\right\rangle\left\langle g_{(1)}, S^{-1} a_{(6)}\right\rangle\left\langle S^{-1} g_{(2)}, a_{(2)} v_{(1)} S a_{(5)}\right\rangle a_{(3)} v_{(2)} S a_{(4)} \\
& =\left\langle g_{(3)}, a_{(1)}\right\rangle\left\langle g_{(1)}, S^{-1} a_{(6)}\right\rangle\left\langle g_{(2)}, a_{(5)} S^{-1} v_{(1)} S^{-1} a_{(2)}\right\rangle a_{(3)} v_{(2)} S a_{(4)} \\
& =\left\langle g, S^{-1} a_{(6)} a_{(5)} S^{-1} v_{(1)} S^{-1} a_{(2)} a_{(1)}\right\rangle a_{(3)} v_{(2)} S a_{(4)} \\
& =\left\langle S^{-1} g, v_{(1)}\right\rangle a_{(1)} v_{(2)} S a_{(2)} \\
& =a \cdot(g \cdot v) .
\end{aligned}
$$

Thus $H$ is a $D(H)$-module under (3). It is clear that (2) is true for $h$ in $H$ or $H^{*}$; since $H$ and $H^{*}$ generate $D(H),(2)$ is also true for $h \in D(H)$. This proves that $H$ is a module algebra of $D(H)$ under the action (3).

We outline a more conceptual proof of Lemma 1 that explains formula (3). For this, we need the following formula for the coproduct of the dual Hopf algebra of $D(H), D(H)^{*}$ (cf. [M]), identifying $D(H)^{*}$ with $H \otimes H^{*}$ :

$$
\Delta(a \otimes g)=\sum\left(a_{(1)} \otimes e_{i}^{*} g_{(1)} e_{j}^{*}\right) \otimes\left(S^{-1}\left(e_{j}\right) a_{(2)} e_{i} \otimes g_{(2)}\right),
$$

where $\left\{e_{i}\right\}$ is a basis of $H$ and $\left\{e_{i}^{*}\right\}$ is its dual basis of $H^{*}$. Now $D(H)^{*}$ is a right module algebra of $D(H)$ under the action $\leftarrow$. It is clear from $(6)$ that $H \subset D(H)^{*}$ is stable under $\leftarrow$, so $H^{o p}$ is a right module algebra of $D(H)$ (here $H^{o p}$ is $H$ with the opposite multiplication). Using (6), it is easy to prove that this right action of $D(H)$ on $H^{o p}$ is given by the formula

$$
b \cdot(g \otimes a)=S^{-1}\left(a_{(2)}\right)(b-g) a_{(1)} .
$$

Now we use the following general fact: if $A^{o p}$ is a right $H$-module algebra with the action $b \cdot h$ for $b \in A, h \in H$, then $A$ is an $H$-module algebra with action $h \cdot b=b \cdot S(h)$. So $H$ is a module algebra of $D(H)$ with the action

$$
(a \otimes f) \cdot b=b \cdot(S(f \otimes a))=\left(a_{(1)} b S\left(a_{(2)}\right)\right) \leftarrow\left(S^{-1} f\right) .
$$

We see that this action is precisely the one defined in (3).

Now we are in the position to state our main theorem.

Theorem 1. If $H$ is a semisimple Hopf algebra over an algebraically closed field $k$ of characteristic 0 , then the action of $D(H)$ given by (3) and the action $\rightarrow$ of $C(H)$ form a commutating pair, i.e., an operator $T \in \operatorname{End}_{k}(H)$ commutes with the action of $D(H)$ if and only if $T$ is in the image of $C(H)$ in $\operatorname{End}_{k}(H)$; and $T \in \operatorname{End}_{k}(H)$ commutes with the action of $C(H)$ if and only if $T$ is the image of $D(H)$ in $\operatorname{End}_{k}(H)$.

Proof. We note that the semisimplicity of $H$ implies that $S^{2}=1$ and $D(H)$ is semisimple ([LR], $[\mathrm{R}])$. The semisimplicity of $H$ also implies that $C(H)$ is a semisimple algebra (cf. [Z]). In particular the images of $D(H)$ and $C(H)$ in $\operatorname{End}_{k}(H)$ are semisimple algebras. Therefore it suffices to prove that $T \in \operatorname{End}_{k}(H)$ commutes with the action of $D(H)$ if and only if $T$ is in the image of $C(H)$. 
Assume $T$ commutes with the action of $D(H)$; we need to prove $T$ is in the image of $C(H)$. We note that $H^{*} \subset D(H)$ acts on $H$ by restriction: this action is just the action "ᄃ" of $H^{*}$ on $H$ twisted by $S^{-1}$. By Lemma 2 below, there exists a unique $v \in H^{*}$ such that

$$
T(b)=v \rightarrow b=\left\langle v, b_{(2)}\right\rangle b_{(1)}
$$

for every $b \in H$.

For $T$ as in (7), $T$ commutes with the action of $H \subset D(H)$ implies that

$$
\left\langle v, a_{(2)} b_{(2)} S a_{(3)}\right\rangle a_{(1)} b_{(1)} S a_{(4)}=\left\langle v, b_{(2)}\right\rangle a_{(1)} b_{(1)} S a_{(2)}
$$

for every $a, b \in H$. Apply the counit map to both sides of (8), we obtain

$$
\left\langle v, a_{(1)} b S\left(a_{(2)}\right)\right\rangle=\langle v, b\rangle \epsilon(a) ;
$$

this further implies that

$$
\langle v, a b\rangle=\left\langle v, a_{(1)} b a_{(3)} S a_{(2)}\right\rangle=\left\langle v, b a_{(2)}\right\rangle \epsilon\left(a_{(1)}\right)=\langle v, b a\rangle .
$$

This proves that $v$ is cocommutative or $v \in C(H)$. Note that in (9), we use the fact that $a_{(2)} S a_{(1)}=\epsilon(a)$ which is true for the Hopf algebras with the property $S^{2}=1$.

Conversely, if $v \in C(H)$, we need to prove that the action " $v \rightarrow$ " commutes with the action of $D(H)$. It is clear that " $v-$ " commutes with the restriction action of $H^{*} \subset D(H)$. Because $v$ is cocommutative,

$$
\begin{aligned}
v \rightarrow(a \cdot b) & =\left\langle v, a_{(2)} b_{(2)} S a_{(3)}\right\rangle a_{(1)} b_{(1)} S a_{(4)} \\
& =\left\langle v, S a_{(3)} a_{(2)} b_{(2)}\right\rangle a_{(1)} b_{(1)} S a_{(4)} \\
& =\left\langle v, b_{(2)}\right\rangle a_{(1)} b_{(1)} S\left(a_{(2)}\right)=a \cdot(v-b) .
\end{aligned}
$$

This proves that " $v \rightarrow$ " commutes with the restriction action of $H \subset D(H)$. Because $D(H)$ is generated by $H^{*} \subset D(H)$ and $H \subset D(H)$, so " $v \longrightarrow$ " commutes with the action of $D(H)$.

Lemma 2. If $T \in \operatorname{End}_{k}(H)$ commutes with the action $<$ of $H^{*}$ on $H$, then there exists $v \in H^{*}$ such that $T(a)=v \rightarrow$ a for all $a \in H$.

Proof. This is a version of the following well-known fact: if $A$ is an associative algebra, $T \in \operatorname{End}_{k}(A)$ commutes with the left multiplication $r_{a}$ for all $a \in A$, then $T$ is a right multiplication for some $b \in A$. To apply this fact, we notice that the transpose action of $\rightarrow$ is the left multiplication of $H^{*}$ on $H^{*}$, while the transpose action of $\leftarrow$ is the right multiplication of $H^{*}$ on $H^{*}$. T commutes with the action — of $H^{*}$ on $H$, implies that $T^{*} \in \operatorname{End}_{k}\left(H^{*}\right)$ commutes with the left multiplications on $H^{*}$. Therefore $T^{*}$ is given by a right multiplication, and therefore there exists $v \in H^{*}$ such that $T(a)=\left(T^{*}\right)^{*}(a)=v \rightarrow a$ for all $a \in H$.

Before giving a corollary of Theorem 1 concerning the dimension of the simple $D(H)$-submodules in $H$, we recall a theorem in [K] (cf. [Z] for an exposition suitable for the discussion here). We assume the conditions in Theorem 1. Since $C(H)$ is semisimple, it is a sum of full matrix algebras $M_{1}, \ldots, M_{s}$. We choose a minimal idempotent $e_{i}$ in $M_{i}$. Then $\operatorname{tr}\left(e_{i}\right)$, the trace of the operator on $H^{*}$ given by $g \mapsto g e_{i}$, is a divisor of $\operatorname{dim}(H)$.

Corollary. Let $H$ be a semisimple Hopf algebra over an algebraically closed field $k$ of characteristic 0 , and let $H$ be the $D(H)$-module defined above. Then the dimension of every simple $D(H)$-submodule in $H$ is a divisor of $\operatorname{dim}(H)$. 
Proof. Let $V_{1}, \ldots, V_{s}$ be the simple $C(H)$-modules correspondent to $M_{1}, \ldots, M_{s}$ respectively. Note that the $C(H)$-action on $H$ is faithful, since this action is the restriction of the $H^{*}$-action "- ". All $V_{i}$ 's appear as submodules in $H$. Because $D(H)$-action and $C(H)$-action form a commuting pair, and both $D(H)$ and $C(H)$ are semisimple, simple $D(H)$-submodules in $H$ and simple $C(H)$-submodules in $H$ are bijectively correspondent. Let $W_{i}(i=1, \ldots, s)$ be the simple $D(H)$ module correspondent to $V_{i}$. As a $D(H) \otimes C(H)$-module, $H$ is isomorphic to $H=\oplus_{i=1}^{s}\left(W_{i} \otimes V_{i}\right)$. Because $e_{i}$ is a minimal idempotent of $M_{i} \subset C(H)$, its trace on $V_{i}$ is 1 , and its trace on $H$ is $\operatorname{dim}\left(W_{i}\right)$ by the above decomposition of $H$. On the other hand, since the $C(H)$-action " - " on $H$ is the transpose action of the action of left multiplication on $H^{*}$, the trace of $e_{i}$ on $H$ is $\operatorname{tr}\left(e_{i}\right)$ above. This proves $\operatorname{dim}\left(W_{i}\right)=\operatorname{tr}\left(e_{i}\right)$. It follows that $\operatorname{dim}\left(W_{i}\right)$ is a divisor of $\operatorname{dim}(H)$.

In the case that $H$ is the group algebra of a finite group $G$ over $\mathbb{C}$, each simple $D(H)$-submodule of $\mathbb{C} G$ is spanned by the elements in a conjugacy class of $G$.

\section{REFERENCES}

[D] V.G.Drinfeld, Quantum Groups, Proc. Int. Cong. Math, Berkeley (1986), 789-820. MR 89f: 17017

[K] G.I.Kats, Certain Arithmetic Properties of Ring Groups, Functional Anal. Appl. 6 (1972), 158-160.

[LR] R.G.Larson and D.E.Radford, Semisimple cosemisimple Hopf Algebras, Amer.J.Math 110 (1988), 381-385. MR 89a:16011

[M] S. Montgomery, Hopf Algebras and Their Actions on Rings, AMS, 1993. MR 94i:16019

[R] D.E. Radford, On the Antipode of a Semisimple Hopf Algebra, J.Algebra 88 (1984), 66-88. MR 85i:16012

[Z] Y.Zhu, Hopf Algebras of Prime Dimension, Intern. Math. Res. Notices No.1 (1994), 53-59. MR 94j:16072

Department of Mathematics, Hong Kong University of Science \& Technology, Clear WATER BAy, HONG KONG 